\section{Is It Possible to Produce Micro-Gravity in Our Own Lab? \\ Sterling Newberry}

Wouldn't it be nice to have some kind of laboratory hood in which gravity is greatly reduced so we could grow more perfect crystals or do biological experiments without having to go into orbit. There was a report in Science last year which hinted that gravity cancellation or modification just might be possible some day ${ }^{1}$, but I doubt that any of our readers noticed it. The title was "Inertia: Does Empty Space Put Up the Resistance?". One has to read this news report carefully to find that physicists have long pondered the connection between the inertial property of mass and the gravitational attraction between masses and that the work quoted here, suggests that once inertia is understood it might be controlled, even canceled. Upon inquiry the editor, Robert Matthews found other physicists believed that the ability to modify inertia could soon be tested experimentally but remarked that "it is a bit too early to be talking about inertia free star ships."

Now I belong to the school of physics which says you don't get something for nothing, so I'm less interested in how to use up energy, or mass, to drive a rocket ship without burning fuel than in the possibility that by manipulating physics, I can shield out the gravitational pull of the earth and moon and sun in a small laboratory space. I don't want to go very deep into the physics of possible methods because no one has proposed how to accomplish this feat of engineering or described a basic physics finding which assures that it can ever be done. I will give you a bibliography so you can go deeper than Robert Matthew's article, if you wish, and mention the names of the physicists who have the daring to look into the basis of the connection between inertial mass and gravitational mass. Most physicists have avoided this question, just taking inertia as an inherent property of matter

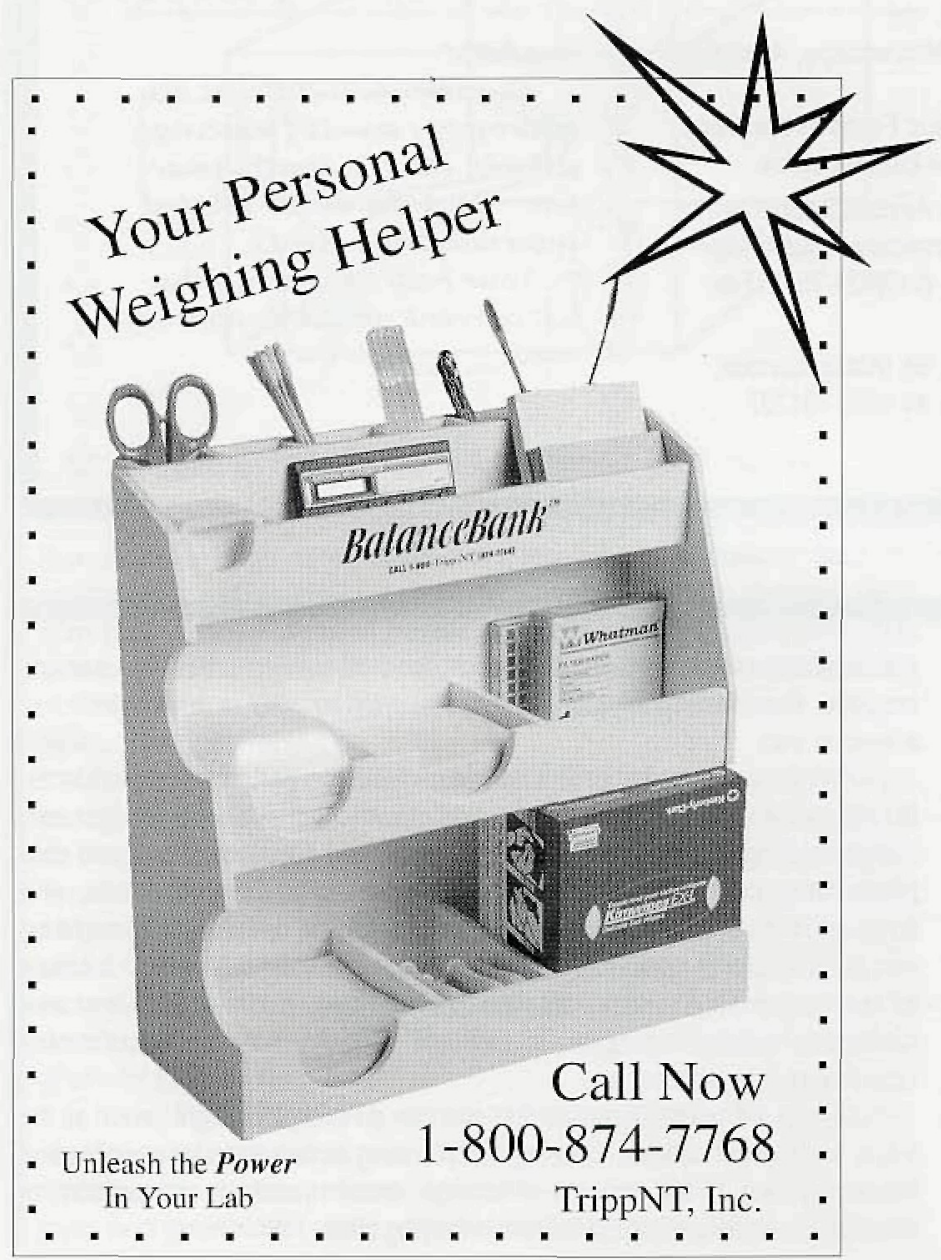

The three physicists are: Bernhard Haisch of Lockheed Palo Alto Research Laboratory, Alfonso Rueda of California State University Long Beach, and H.E. Puthoff of Institute for Advanced Studies at Austin. They consider inertia to be similar to Van der Waals and Casimir forces in its origin, namely from the interactions of the fundamental particle of matter called partons. I prefer to quote a few of their words directly. In one of their papers ${ }^{2}$ they say "we thus propose the interpretation that inertia is an electromagnetic resistance arising from the known spectral distortion of the zero-point field in accelerated frames" and in the next section "Recently one of $\mathrm{us}^{3}$ analyzed a hypothesis of Sakharov" that Newtonian Gravity could be interpreted as a Van der Waals type force induced by the electromagnetic fluctuations of the vacuum, the so-called zero-point fluctuations or zero-point field". What they have added is mathematical formulation which shows resistive forces under acceleration but not at uniform velocity thus giving a possible mathematical basis for Newton's laws of motion. No one previously has derived inertia from a more basic concept, all others including Einstein have taken inertia as a given fact of nature. Many did, however wonder about the cause of inertia, going as far back as Ernst Mach in 1872 (1). Furthermore I should point out that physics is in turmoil as we approach closer to understanding of the nucleus and thus other people are revisiting many of the older questions including the ones Haisch et. al. are examining. It appears to me that through different approaches all of physics is converging on to the same fundamental concepts ${ }^{5}$.

Without knowing the specific physics, what can we predict about the likely form a laboratory micro-gravity hood might assume? First off we can expect that the walls of the hood will not have to be very strong since the ordinary gravitational field is quite weak. We can further expect that the presence of atmosphere and or manipulative apparatus inside the micro-gravity space will not add appreciably to the gravitational component inside compared to that which we exclude from the rest of the universe. If one wants to do experiments in vacuum also, then the walls will have to support an additional 14.7 pounds per square inch. Not knowing the mechanism of gravity shielding, we cannot exclude the possibility that substantial power may be required to sustain the shield and with expenditure of power we must expect the likelihood of cooling problems.

What ever the mechanism of shielding, it will likely involve some kind of solid state or multilayered structure which has very small wave guide or quantum well structures. Thus we would expect such a layered structure to be attached to the metal walls so as to provide good thermal contact. We will be fortunate indeed if this shielding layer does not have to operate at very low temperatures.

The introduction of samples will probably require the construction of a gravity lock, whose shield can be turned on and off as required, so that one sample may be retrieved without interrupting the progress of other samples.

For the question of gravity leakage through interruptions of the shielding we have no hints yet. Can we have control rods, electrical feed-thrus and windows in the walls? We may be faced with programmed internal manipulators and transmission of images from interior sensors which we can communicate with only at very low repetition rates and/or with difficult frequency regions of the electromagnetic spectrum such as the " $T$ " waves".

Probably at this point the reader will agree that if we ever have the microgravity hood we will look back with longing for the simple days of ultra high vacuum and electron microscope design.

1. "Inertia: Does Empty Space Put Up the Resistance?" report by Robert Matthews ed., Science vol 263 pgs 612-13, February 4, 1994.

2. "Inertia as a zero-point-field Lorentz force" Haisch, Rueda and Puthoff, Physical Rev. A, vol 49 \#2, pgs 678-94, February 1994.

3. "Gravity as a zero-point-fluctuation force" H. E. Puthoff, Physical Rev. A vol 39 \#5, pgs. 2333-42, March 1, 1989.

4. A.D. Sakharov, Dokl. Akad. Nauk. SSSR [Sov. Phys.-Dokl. 12, 1040 (1968)].

5 "The New Physics" Edited by Paul Davies, Especially chapter 18 by Abdus Salam on overview of particle physics, Cambridge U. Press, 1989.

6. "Scenes From a Marriage of Optics and Electronics" a meeting report by Robert F. Service, Science vol 268 pgs 1702-3, June 23, 1995. 


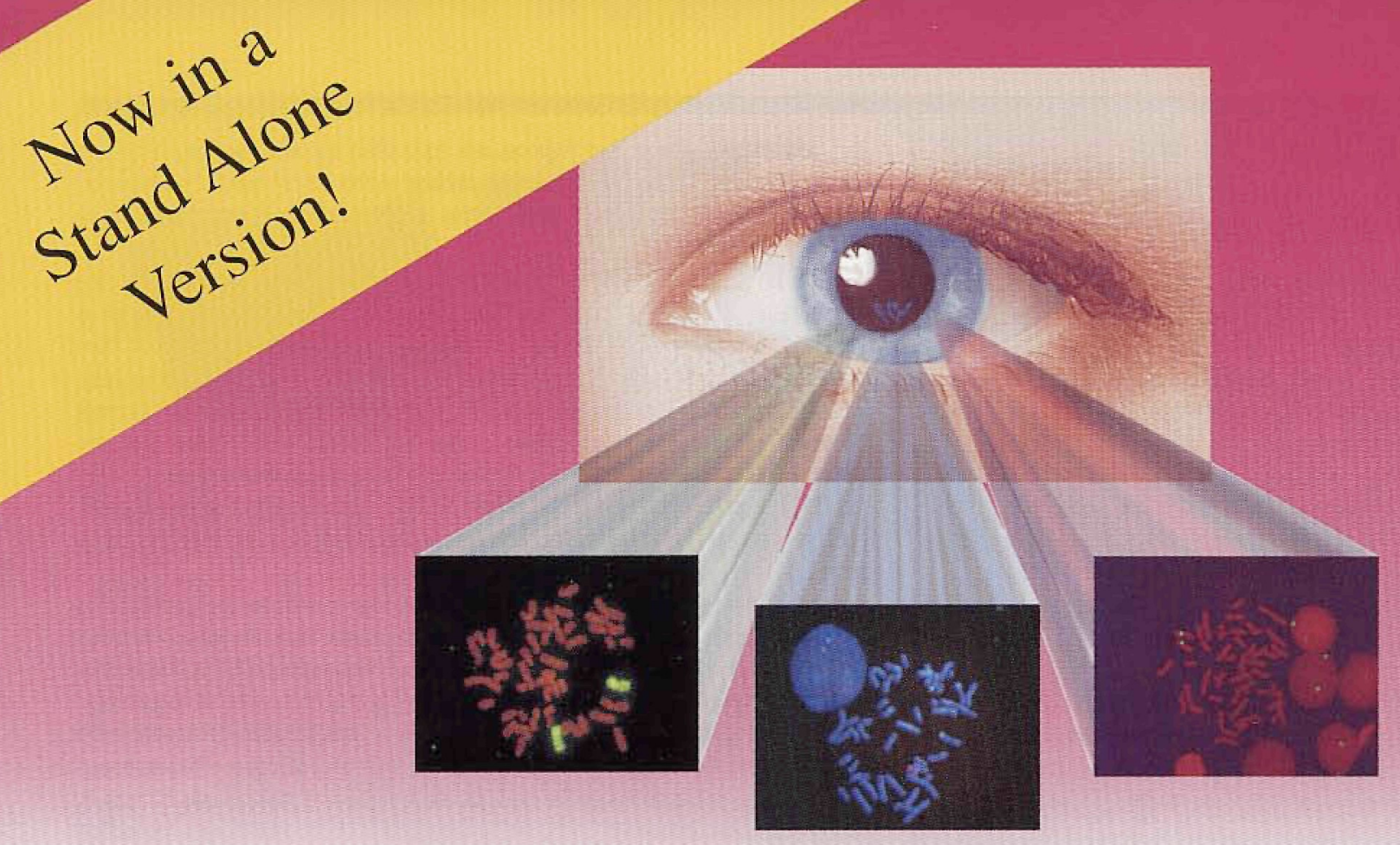

\section{There's More to Our New Camera than Meets the Eye}

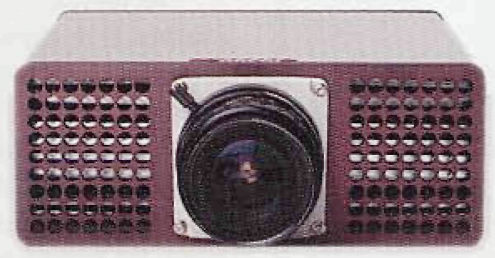

\section{Introducing our NEW Cooled 3CCD High Resolution, Color, Low Light Camera}

Capture true color, high resolution, low light images and output them in just seconds!

- Dramatic Improvement in Picture Quality

- Capture Smaller FISH Probes

- Built-in Sharpening Filter

- Ten Fold Increase in Sensitivity

- Easy to Operate Software Controls

- Labeling Ability

Just when you think you've seen it all - a camera that stretches the limits of what you CAN see - Oncor advances its leadership in imaging systems technology with its new, cooled 3CCD Color Camera System.

Oncor's innovative color camera system not only operates accurately at very low fluorescent light levels, it also provides exceptional imaging for brightfield microscopy. This ability offers the user a greater capacity to address a wider range of applications.
For superior results, Oncor's total-systems approach maximizes performance by using Oncor's DNA and reagents in Fluorescence in Situ Hybridization (FISH) applications.

Call us at 1-800-77-0NCOR.

You've got to see it ! It's cool !

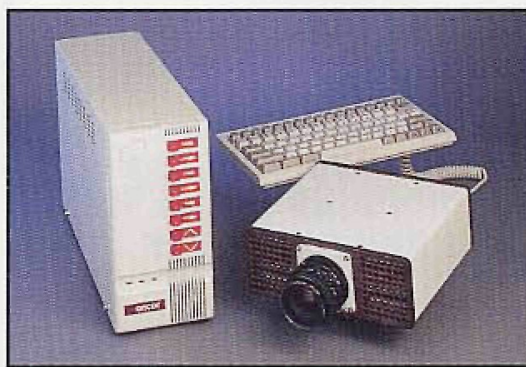

209 Peny Parkway, Gaitbersburg, $M D 20877$ USA 1-800-77-ONCOR • 14X 301-926-6129

* For research use only. Not for use in diagnostic procedures. 